\title{
Editorial
}

Miguel González, Manuel Molina and Inés M. del Puerto*

\section{5th International Workshop on Branching Processes and Their Applications (IWBPA 2021)}

https://doi.org/10.1515/eqc-2021-2001

The 5th International Workshop on Branching Processes and Their Applications (IWBPA 2021) was held on 6th, 8th, 13th, 15th, 20th and 22nd April 2021. It was the last in a series of meetings held every three years in Badajoz (Spain). Due to the COVID-19 pandemic, the IWBPA 2021 took place virtually using video conferencing tools. The 2021 year's conference continued the tradition of the previous meetings in facilitating the exchange of research ideas in this field and related processes. Let us recall that the First World Congress on Branching Processes was held in Varna (Bulgaria) in 1993 to celebrate the 150th anniversary years of branching processes. International meetings on branching processes took place in Gothenburg (Sweden) in 2005 and in Luminy (France) in 2007 and 2011. Branching processes research has been presented at the Summer Schools held biannually in Bulgaria since 2012 and annually in China since 2015. The IWBPA 2021 meeting was the fifth in the series of IWBPAs promoted and organized since 2009 by the research group Branching Processes and their Applications at the Department of Mathematics of the University of Extremadura, Spain, and scientifically sponsored by the Spanish Society for Statistics and Operations Research (SEIO). There were 145 participants and 58 speakers from 22 countries who contributed to the success of the workshop. The presentations at the workshop maintained a healthy balance between the theoretical and practical aspects of branching processes. The speakers articulated the fact that this research area is very active and produces interesting results. The conference program and talks are available on the website (https://sites.google.com/ view/iwbpa21-branching-unex).

This special issue, dedicated to the Workshop, contains six selected papers. S. Sagitov [5] extends the convergence of a properly scaled critical Galton-Watson process to a continuous state critical branching process, as the number of initial individuals tends to infinity, by allowing for overlapping generations and considering a wide class of population counts. G. Alsmeyer [2] considers linear fractional Galton-Watson branching processes in independent and identically distributed random environment and their relationships with random difference equations given by the evolution of the random parameters of their linear fractional marginals. V. Afanasyev [1] establishes limit theorems for a strongly supercritical branching process in random environment with immigration stopped at a fixed distant time. Y. Wang and Q. Liu [6] present a short survey about asymptotic properties of a supercritical branching process with immigration in a stationary and ergodic or independent and identically distributed random environment. D. Atanasov, V. Stoimenova and N. Yanev [3] propose to model COVID-19 outbreak dynamics using a class of two-type branching processes. I. Rahimov [4] summarizes and discusses important known results for homogeneous branching processes with non-homogeneous immigration.

\footnotetext{
*Corresponding author: Inés M. del Puerto, Department of Mathematics, Faculty of Sciences, University of Extremadura, Avda. De Elvas, s/n, 06006 Badajoz, Spain, e-mail: idelpuerto@unex.es. https://orcid.org/0000-0002-1034-2480

Miguel González, Manuel Molina, Department of Mathematics, Faculty of Sciences, University of Extremadura, Avda. De Elvas, s/n, 06006 Badajoz, Spain, e-mail: mvelasco@unex.es, mmolina@unex.es. https://orcid.org/0000-0001-7481-6561, https://orcid.org/0000-0002-7919-5298
} 
We would like to acknowledge the reviewers for their competent and efficient work. We appreciate the Editor-in-Chief's essential assistance as well as the help of De Gruyter's professionals during the entire process of publishing this Special Issue. Let us also point out that the Editorial Board of Stochastics and Quality Control with the strong support of De Gruyter, has been working in establishing this journal as an avenue for publishing research results in the area of branching processes and their applications.

Badajoz, December 17, 2021

M. González, M. Molina and I. del Puerto (Guest Editors)

\section{References}

[1] V. Afanasyev, Limit theorems for a strongly supercritical branching process with immigration in random environment, Stoch. Qual. Control 36 (2021), no. 2, 129-143.

[2] G. Alsmeyer, Linear fractional Galton-Watson processes in random environment and perpetuities, Stoch. Qual. Control 36 (2021), no. 2, 111-127.

[3] D. Atanasov, V. Stoimenova and N. Yanev, Branching process modelling of COVID-19 pandemic including immunity and vaccination, Stoch. Qual. Control 36 (2021), no. 2, 157-164.

[4] I. Rahimov, Homogeneous branching processes with non-homogeneous immigration, Stoch. Qual. Control 36 (2021), no. 2, 165-183.

[5] S. Sagitov, Critical Galton-Watson processes with overlapping generations, Stoch. Qual. Control 36 (2021), no. 1, 87-110.

[6] Y. Wang and Q. Liu, Asymptotic properties of a supercritical branching process with immigration in a random environment, Stoch. Qual. Control 36 (2021), no. 2, 145-155. 